\title{
Prediction of Nipple and Areola Complex Invasion in Breast Cancer Patients. Clinical and Pathological Study of Surgical Specimens
}

\author{
Mohammed H. Goda1, Mostafa Abdel Rahman', Emadeldin R. Matar2* \\ ${ }^{1}$ Faculty of Medicine, Ain Shams University, Cairo, Egypt \\ ${ }^{2}$ Faculty of Medicine, Al Azhar University, Cairo, Egypt \\ Email: *Emad_rshde61@hotmail.com
}

How to cite this paper: Goda, M.H., Rahman, M.A. and Matar, E.R. (2021) Prediction of Nipple and Areola Complex Invasion in Breast Cancer Patients. Clinical and Pathological Study of Surgical Specimens. Open Journal of Pathology, 11, 59-78.

https://doi.org/10.4236/ojpathology.2021.1 $\underline{13006}$

Received: April 18, 2021

Accepted: May 29, 2021

Published: June 1, 2021

Copyright $\odot 2021$ by author(s) and Scientific Research Publishing Inc. This work is licensed under the Creative Commons Attribution International License (CC BY 4.0).

http://creativecommons.org/licenses/by/4.0/

\section{(c) (i) Open Access}

\begin{abstract}
Breast conservation surgery (BCS) and nipple-areola-sparing (NAS) mastectomy have been recognized as two milestones in this period. This study included 60 Egyptian female patients with breast cancer, all of them were subjected to modified radical mastectomy operation. Methods: This study included female patients $>18$ years old who have breast cancer with healthy looking non invaded skin of nipple and areola and excluded patients $<18$ years old, patients unfit for surger, patients previously subjected to chemo or radiotherapy for breast cancer. We peformed clinical examination of 60 patients with breast cancer. We studied the relevant factors that affect NAC invasion such as patient's age, menstrual state, family history, tumor size, tumor location (central vs peripheral), tumor to nipple distance, lymphovascular invasion of NAC, lymph node metastasis, histological tumor type, tumor stage, multifocal/multicentric tumors and (ER, PR, HER2) status. Result: In our study, we have shown that NAC invasion is strongly associated with: 1) Nipple retraction as a patient's complaint; 2) Tumor site; 3) Tumor-nipple-distance $\leq 4 \mathrm{~cm}$; 4) Multifocal/multicentric tumor; 5) Tumor grade (grade III tumors); 6) Positive lymph node invasion; 7) ER and PR receptors negativity; 8) HER2 positivity. This helps in preoperative planning for selecting patients for NAS mastectomy. Conclusion: The ideal patients for NAS mastectomy are with these criteria: 1) Clinically normal nipple areola complex; 2) Distance from the tumor to the nipple is $>4 \mathrm{~cm}$; 3) No multifocal/multicentric tumor; 4) Absence of lymph node invasion; 5) Tumor grade (grade I, II); 6) Peripheral not central tumor; 7) No sub-areolar lymphovascular invasion (LVI); 8) ER receptor positive; 9) PR receptor positive; 10) HER2 negative.
\end{abstract}




\section{Keywords}

Nipple and Areola Invasion, Breast Cancer, Clinical and Pathological

\section{Introduction}

Breast cancer remains the most frequently diagnosed cancer among women. Surgical techniques have evolved from radical mastectomy to less invasive and cosmetically acceptable surgical approach in recent years [1].

Breast conservation surgery (BCS) and nipple-areola-sparing (NAS) mastectomy have been recognized as two milestones in this period [2]. Oncoplastic surgery involves attention to tumor oncology as well as breast aesthetics. It has become popular amongst breast surgeons, combining oncologic principles with plastic surgical techniques [2] [3].

NAS mastectomy entails removal of all breast tissues with preservation of the entire skin of the breast and the nipple-areola complex (NAC) [4].

NAS mastectomy gained great popularity among breast cancer patients, either for treatment or risk reduction. The prophylactic treatment is indicated in women with BRCA1/BRCA2 mutations, personal or family history of breast cancer and severe fibrocystic disease with a strong family history of cancer [5].

\section{Aim of the Work}

The aim of this work is to study the clinical and pathological criteria upon which a decision can be made for nipple areola sparing mastectomy in breast cancer patients.

\section{Patients and Methods}

This study was carried out in the departments of general surgery and oncology unit, through the period from January 2017 to July 2020.

The study included 60 Egyptian female patients with breast cancer all of them were subjected to modified radical mastectomy operation.

\subsection{Patient's Selection}

1) Inclusion criteria:

- Female patient $>18$ years old.

- Breast cancer patients with healthy looking non invaded skin of nipple and areola.

- Patient $<18$ years old.

- Patients accept participation.

- Patients fit for surgery.

- Patients not previously subjected to chemo or radiotherapy for breast cancer.

- No inflammatory breast cancer cases.

- Patients without skin changes involving nipple areola complex. 
2) Exclusion criteria:

- Patient $<18$ years old.

- Patients' refusal.

- Patients unfit for surgery.

- Patients previously subjected to chemo or radiotherapy for breast cancer.

- Inflammatory breast cancer cases.

- Patients who had skin changes involving nipple areola complex.

\subsection{Pre-Operative}

\section{1) History taking:}

The history included:

a) Personal history:

As regard name, age, marital status including number of children, occupation and any special habits.

\section{b) Complaint:}

Painless lump, Painful lump, Pain with no lump, nipple discharge, nipple retraction, Skin changes and axillary lump with its duration.

c) Present history: as regard

- Assessment of breast mass e.g. onset, course, duration, number, site, size, painful or not, associated with nipple discharge or not, ...

- Assessment of any axillary swelling(s) e.g. onset, course, duration, number, site, size, ...

- Assessment of skin and nipple changes e.g. nipple discharge (especially bloody one), nipple erosion, skin nodules, ... (refer to exclusion criteria)

- Assessment of any symptoms suggestive of metastases e.g. bone-aches, jaundice, hemoptysis, neurological deficit, ...

- Review of sympoms related to other systems e.g. gastro-intestinal and abdominal symptoms, urological symptoms, genital symptoms, cardio vascular symptoms, respiratory symptoms, nervous system, musculoskeletal system and endocrine system.

\section{d) Menstrual history:}

As regard age at menarche, regularity of the menstrual cycle, age at the first childbearing, age at menopause (for post-menopausal women), and use of contraceptive pills.

e) Family history:

History of breast cancer especially in relatives (sister, mother or daughter).

\section{f) Past history:}

History of previous operations, allergy and sensitivity, diseases and drugs.

\section{2) Physical examination}

a) General examination:

As regard vital signs (pulse, blood pressure, temperature, and respiration), body built, general appearance and systemic examination of all body systems with special concern looking for signs of metastases. 


\section{b) Local examination:}

Local examination of the normal breast and axilla was done first before examination of the affected side.

- Examination of any breast mass assessing tenderness, hotness, site, size, shape, surface, number, consistency, edge, relation to underlying structures (fixation to pectoral muscles or chest wall), and relation to overlying skin for evidence of malignant invasion (skin ulceration, peau d'orange, fixity, etc.).

- Assessment of the depth of the mass in breast tissue either, superficial or deep.

- Examination of the axillary lymph nodes assessing number, size, shape, surface, consistency, matting to each other and fixing underlying structures and which group(s) of lymph node is/are affected.

\section{3) Pre-operative investigations:}

a) Laboratory investigations:

- Complete blood count (CBC).

- Bleeding profile (PT, PTT and INR).

- Liver function tests (total and direct bilirubin, serum albumin, ALT and AST).

- Kidney function tests (blood urea and creatinine).

- Random blood sugar.

- Hepatitis markers.

\section{b) Mammography:}

All patients were subjected to bilateral soft tissue mammography, where cranio-caudal and oblique views of both breasts were obtained.

\section{c) Breast ultrasonography:}

It was done as a complementary imaging technique to mammography to confirm diagnosis, differentiate cystic swellings from solid ones and help take U/S-guided FNAC from suspicious lesions. Now, in many centers it is superior to mammography and the investigation of choice.

\section{d) Fine needle aspiration cytology (FNAC):}

It was done as an outpatient technique and was done U/S-guided.

\section{e) Excisional biopsy:}

It was done when FNAC was not conclusive to diagnose malignancy.

f) Investigations to rule out metastasis:

- Chest x-ray.

- Pelvi-abdominal U/S.

g) ECG and medical fitness for surgery: for patients over 40 years.

h) Informed consent for mastectomy was signed by all patients after detailed explanation to them.

\subsection{Operative Work}

After doing all investigations and confirming the stage of the disease and fitness of the patients for surgery, patients were prepared to modified radical mastectomy. 


\subsection{Post-Operative}

The specimens sent to the pathology department. Breast specimen were examined by standard hematoxylin and eosin stains under light microscopy. Tissue just underlying nipple-areola complex were examined for evidence of malignancy. All specimens were examined by a single expert pathologist to search for malignancy in the subareolar tissues.

Histopathology for:

Diagnosis:

Histological Type:

Tumor Grade:

Lymph vascular invasion of subareolar complex:

Lymph Node Status:

Number of lymph node in each level:

Total Number: +Ve/Total

Hormonal status.

Post-operative care:

1) Monitoring of the vital signs of the patients every 6 hours and the amount of the drain every 12 hours.

2) Patients were encouraged for early mobilization from bed after 6 hours and started oral intake.

3) Post-operative analgesia was given in the form of diclofenac sodium $75 \mathrm{mg}$ IM every 12 hours.

4) Follow up of any post-operative complications such as wound infection, seroma, hematoma and numbness along the inner aspect of the arm.

5) The suction drain was removed when the serous discharge decreased to less than $30 \mathrm{ml}$ in 24 hours.

6) The sutures of the wound were removed from days $10-12$.

7) The patients were referred to the oncology department with the final pathology report to complete their post-operative radiotherapy, chemotherapy and/or hormonal therapy.

8) Follow up of patients for 2 years.

\section{Statistical Analysis}

All data were collected, tabulated and statistically analyzed using Statistical package for the social sciences (SPSS). 22.0 for windows (SPSS Inc., Chicago, IL, USA).

\section{Results}

Table 1: Mean age of breast cancer patients without NAC invasion was non-significantly higher than mean age of breast cancer patients with NAC invasion (Mean: 51.26 vs 49.90 years, p-value $=0.741$ ), also non-significant association between age group and NAC invasion where $26 \%$ of patients without NAC invasion was age less than 40 years versus $18.2 \%$ in patients with NAC invasion 
Table 1. Demographic data of the studied groups.

\begin{tabular}{|c|c|c|c|c|c|c|}
\hline \multirow[b]{2}{*}{ Demographic data } & \multicolumn{4}{|c|}{ NAC invasion } & \multirow[b]{2}{*}{ Test } & \multirow{2}{*}{$\begin{array}{c}\text { p-value } \\
\text { (Sig.) }\end{array}$} \\
\hline & \multicolumn{2}{|c|}{$\begin{array}{l}\text { Absent } \\
(\mathrm{N}=50)\end{array}$} & \multicolumn{2}{|c|}{$\begin{array}{l}\text { Present } \\
(\mathrm{N}=10)\end{array}$} & & \\
\hline \multicolumn{7}{|l|}{ Age (years) } \\
\hline Mean \pm SD & \multicolumn{2}{|c|}{$51.26 \pm 12.56$} & \multicolumn{2}{|c|}{$49.90 \pm 10.26$} & $0.332^{*}$ & $\begin{array}{l}0.741 \\
(\mathrm{NS})\end{array}$ \\
\hline$<40$ years & 13 & $26 \%$ & 2 & $18.2 \%$ & & \\
\hline 40 - 60 years & 20 & $40 \%$ & 8 & $72.7 \%$ & $4.224^{\ddagger}$ & $\begin{array}{c}0.121 \\
\text { (NS) }\end{array}$ \\
\hline$>60$ years & 17 & $34 \%$ & 1 & $9.1 \%$ & & \\
\hline \multicolumn{7}{|l|}{ Menstrual state } \\
\hline Premenopausal & 32 & $36 \%$ & 6 & $\begin{array}{l}45.5 \% \\
54.5 \%\end{array}$ & $0.343^{\ddagger}$ & $\begin{array}{l}0.558 \\
(\mathrm{NS})\end{array}$ \\
\hline \multicolumn{7}{|l|}{ Family History } \\
\hline Negative & 13 & $26 \%$ & 8 & $\begin{array}{l}72.7 \% \\
27.3 \%\end{array}$ & $0.008^{\ddagger}$ & $\begin{array}{l}1.000 \\
\text { (NS) }\end{array}$ \\
\hline \multicolumn{7}{|l|}{$\underline{\text { Parity }}$} \\
\hline Nullipara & 37 & $74 \%$ & 3 & $\begin{array}{l}72.7 \% \\
27.3 \%\end{array}$ & $0.008^{\ddagger}$ & $\begin{array}{l}1.000 \\
(\mathrm{NS})\end{array}$ \\
\hline \multicolumn{7}{|l|}{ Lactation } \\
\hline NO & 37 & $74 \%$ & 8 & $72.7 \%$ & $0.008^{\ddagger}$ & $\begin{array}{l}1.000 \\
\text { (NS) }\end{array}$ \\
\hline
\end{tabular}

${ }^{*}$ Independent samples Student's t-test. ${ }^{\ddagger}$ Chi-square test. $\mathrm{p}<0.05$ is significant. Sig.: Significance.

(p-value $=0.121)$, on other hand there's non-significant association between menstrual state and NAC invasion, where $64 \%$ of patients without NAC invasion was postmenopausal vs $54.5 \%$ in patients with NAC invasion ( $\mathrm{p}$-value = 0.558). 13 out of 50 patients (26\%) without NAC invasion had positive family history of breast cancer versus 3 out of 11 patients (27.3\%) with NAC invasion $(\mathrm{p}=1.000)$, So there's non-significant association between family history and NAC invasion. There is a significant association between patient's complaint (nipple retraction) and NAC invasion where $2 \%$ of patients without NAC invasion complaint from nipple retraction versus $36.7 \%$ of patients with NAC invasion (p-value $=0.001) .27$ out of 50 patients $(54 \%)$ without NAC invasion had right breast cancer versus 5 out of 10 patients $(50 \%)$ with NAC invasion $(\mathrm{p}=$ 0.078). Mean breast size in patients without NAC invasion was non-significantly lower than mean breast size in patients with NAC invasion (Mean: 42.48 vs 43.63, p-value $=0.186) .100 \%$ of patients without NAC invasion had unilateral breast cancer versus $90 \%$ of patients with NAC invasion ( $p$-value $=0.167)$. There is a significant association between focality and NAC invasion where $0 \%$ of patients without NAC invasion had multifocal breast cancer versus $81.8 \%$ of pa- 
tients with NAC invasion (p-value < 0.001) (Table 2). There is a significant association between tumor site (centrally located tumors) and NAC invasion where $0 \%$ of patients without NAC invasion had central tumor versus $30 \%$ of patients with NAC invasion ( $\mathrm{p}$-value $<0.001$ ). There is a significant association between tumor site (centrally located tumors) and NAC invasion where $0 \%$ of patients without NAC invasion had central tumor versus $30 \%$ of patients with NAC invasion ( $p$-value $<0.001$ ). Mean clinical size of tumor in patients without NAC invasion was non-significantly higher than clinical size of tumor in patients with NAC invasion (Mean: 3.30 vs $2.85 \mathrm{~cm}$, p-value $=0.401$ ). Mean ultrasound size of tumor in patients without NAC invasion was non-significantly higher than clinical size of tumor in patients with NAC invasion (Mean: 2.60 vs 2.57, $\mathrm{p}$-value $=0.838)($ Table 3 and Table 4$)$.

Table 5 and Table 6: 39 out of 50 patients (78\%) without NAC invasion had T2 breast cancer versus 10 out of 11 patients (90.9\%) with NAC invasion ( $\mathrm{p}=$ 0.606). There is a significant association between clinical $N$ stage and NAC invasion where $20 \%$ of patients without NAC invasion had N3 versus $63.6 \%$ of patients with NAC invasion ( $\mathrm{p}$-value $=0.014$ ). Mean distance from mass to nipple in patients without NAC invasion was significantly higher than in patients with NAC invasion (Mean: 7.24 vs $3.15 \mathrm{~cm}$, p-value < 0.001), so there's significant association between distance from mass to nipple and NAC invasion. 33 out of 50 masses $(66 \%)$ without NAC invasion were superficial masses versus 14 out of 20 masses $(70 \%)$ with NAC invasion $(\mathrm{p}=0.748)$.

Table 7 \& Figure 1: Optimum cutoff of distance from mass to nipple as a predictor for NAC invasion in breast carcinoma was $\leq 4 \mathrm{~cm}$. So, $100 \%$ of patients with distance $>4 \mathrm{~cm}$, can be expected that they haven't NAC invasion. In other hand no patients with distance $>4 \mathrm{~cm}$, can be expected that they have NAC invasion while $85 \%$ of patients with distance $\leq 4 \mathrm{~cm}$, can be expected that they have NAC invasion in other hand $15 \%$ of patients with distance $\leq 4 \mathrm{~cm}$, can be expected that they haven't NAC invasion, so we conclude that this cutoff is optimum to exclude NAC invasion (patients with distance $>4 \mathrm{~cm}$ ) rather than conclude NAC invasion (patients with distance $\leq 4 \mathrm{~cm}$ ). 32 out of 50 patients (64\%) without NAC invasion underwent core biopsy versus 7 out of 11 patients $(63.6 \%)$ with NAC invasion ( $\mathrm{p}$-value $=0.306) .84 \%$ of patients without NAC invasion had infiltrating duct carcinoma versus $90.9 \%$ of patients with NAC invasion ( $\mathrm{p}$-value $=0.624)$. There is a significant association between histological grade and NAC invasion where $40 \%$ of patients without NAC invasion was grade III versus $90.9 \%$ of patients with NAC invasion ( $\mathrm{p}$-value $=0.002$ ). $60 \%$ of patients without NAC invasion were node positive versus $100 \%$ of patients with NAC invasion $(p$-value $=0.011)$. Number of positive lymph node in patients with NAC invasion was significantly higher than patients without NAC invasion (Mean: 13.72 vs 4.98 , p-value $=0.001$ ). There is non-significant difference between patients without and with NAC invasion as regard number of dissected lymph nodes (Mean: 17.98 vs 18.45, p-value $=0.707$ ) (Table 8). 39 out of 50 patients $(78 \%)$ without NAC invasion had pT2 breast cancer versus 10 out of 11 
Table 2. Clinical data of the studied groups.

\begin{tabular}{|c|c|c|c|c|c|c|}
\hline \multirow[b]{2}{*}{ Clinical data } & \multicolumn{4}{|c|}{ NAC invasion } & \multirow[b]{2}{*}{ Test } & \multirow[b]{2}{*}{$\begin{array}{l}\text { p-value } \\
\text { (Sig.) }\end{array}$} \\
\hline & \multicolumn{2}{|c|}{$\begin{array}{c}\text { Absent } \\
(\mathrm{N}=50)\end{array}$} & \multicolumn{2}{|c|}{$\begin{array}{l}\text { Present } \\
(\mathrm{N}=11)\end{array}$} & & \\
\hline \multicolumn{7}{|l|}{ Patient's complaint } \\
\hline \multicolumn{7}{|l|}{$\underline{\text { Painless lump }}$} \\
\hline $\begin{array}{l}\text { Absent } \\
\text { Present }\end{array}$ & 32 & $\begin{array}{l}36 \% \\
64 \%\end{array}$ & 4 & $\begin{array}{l}63.6 \% \\
36.4 \%\end{array}$ & $2.847^{\ddagger}$ & $\begin{array}{l}0.174 \\
(\mathrm{NS})\end{array}$ \\
\hline $\begin{array}{l}\text { Painful lump } \\
\text { Absent } \\
\text { Present }\end{array}$ & 36 & $\begin{array}{l}72 \% \\
28 \%\end{array}$ & 9 & $\begin{array}{l}81.8 \% \\
18.2 \%\end{array}$ & $0.449^{\ddagger}$ & $\begin{array}{l}0.711 \\
(\mathrm{NS})\end{array}$ \\
\hline $\begin{array}{l}\text { Axillary lump } \\
\text { Absent } \\
\text { Present }\end{array}$ & 48 & $\begin{array}{l}96 \% \\
4 \%\end{array}$ & 11 & $\begin{array}{c}100 \% \\
0 \%\end{array}$ & $0.455^{\ddagger}$ & $\begin{array}{l}0.500 \\
(\mathrm{NS})\end{array}$ \\
\hline $\begin{array}{c}\text { Nipple discharge } \\
\text { Absent } \\
\text { Present }\end{array}$ & 49 & $\begin{array}{l}98 \% \\
2 \%\end{array}$ & 11 & $\begin{array}{c}100 \% \\
0 \%\end{array}$ & $0.224^{\ddagger}$ & $\begin{array}{l}1.000 \\
(\mathrm{NS})\end{array}$ \\
\hline $\begin{array}{c}\text { Bleeding per nipple } \\
\text { Absent } \\
\text { Present }\end{array}$ & 50 & $\begin{array}{c}100 \% \\
0 \%\end{array}$ & 10 & $\begin{array}{l}90.9 \% \\
9.1 \%\end{array}$ & $4.621^{*}$ & $\begin{array}{l}0.180 \\
(\mathrm{NS})\end{array}$ \\
\hline $\begin{array}{c}\text { Nipple retraction } \\
\text { Absent } \\
\text { Present }\end{array}$ & 49 & $\begin{array}{l}98 \% \\
2 \%\end{array}$ & 4 & $\begin{array}{l}63.6 \% \\
36.7 \%\end{array}$ & 14.149 & $\begin{array}{c}0.003 \\
(\mathrm{~S})\end{array}$ \\
\hline \multicolumn{7}{|l|}{$\underline{\text { Affected breast }}$} \\
\hline $\begin{array}{l}\text { Right breast } \\
\text { Left breast }\end{array}$ & 27 & $\begin{array}{l}54 \% \\
46 \%\end{array}$ & 4 & $\begin{array}{l}50 \% \\
40 \% \\
10 \%\end{array}$ & $5.092^{\ddagger}$ & $\begin{array}{l}0.078 \\
(\mathrm{NS})\end{array}$ \\
\hline \multicolumn{7}{|l|}{ Breast size (bra size) } \\
\hline $\begin{array}{c}\text { Mean } \pm \text { SD } \\
\text { Median (Range) }\end{array}$ & & $\begin{array}{l}2.47 \\
46)\end{array}$ & & $\begin{array}{l}2.65 \\
-46)\end{array}$ & $-1.321 \bullet$ & $\begin{array}{l}0.186 \\
(\mathrm{NS})\end{array}$ \\
\hline \multicolumn{7}{|l|}{ Bilaterality } \\
\hline Yes & 50 & $100 \%$ & 9 & $\begin{array}{l}90 \% \\
10 \%\end{array}$ & $5.085^{\ddagger}$ & $\begin{array}{l}0.167 \\
(\mathrm{NS})\end{array}$ \\
\hline \multicolumn{7}{|l|}{$\underline{\text { Focality }}$} \\
\hline Multifocal & 50 & $100 \%$ & 2 & $\begin{array}{l}18.2 \% \\
81.8 \%\end{array}$ & $47.990^{\ddagger}$ & $\begin{array}{c}<0.001 \\
(\mathrm{HS})\end{array}$ \\
\hline
\end{tabular}




\begin{tabular}{|c|c|c|}
\hline Tumor site & \multicolumn{2}{|c|}{$\begin{array}{c}\text { All masses } \\
(\mathrm{N}=70)\end{array}$} \\
\hline Non palpable & 2 & $2.9 \%$ \\
\hline Upper outer $1 / 4$ & 42 & $60 \%$ \\
\hline Upper inner $1 / 4$ & 7 & $10 \%$ \\
\hline Lower outer $1 / 4$ & 6 & $8.6 \%$ \\
\hline Lower inner $1 / 4$ & 5 & $7.1 \%$ \\
\hline Central & 6 & $8.6 \%$ \\
\hline Axillary tail & 2 & $2.9 \%$ \\
\hline Variables & & \\
\hline \multicolumn{3}{|c|}{$\underline{\text { Distance from mass to nipple }}$} \\
\hline Mean \pm SD & & \\
\hline Median (Range) & & \\
\hline \multicolumn{3}{|l|}{ Depth of the mass } \\
\hline Superficial & 47 & $67.1 \%$ \\
\hline Deep & 23 & $32.9 \%$ \\
\hline
\end{tabular}

-Mann Whitney U test. ${ }^{\ddagger}$ Chi-square test. $\mathrm{p}<0.05$ is significant. Sig.: Significance.

Table 3. Site of the tumor of the studied groups.

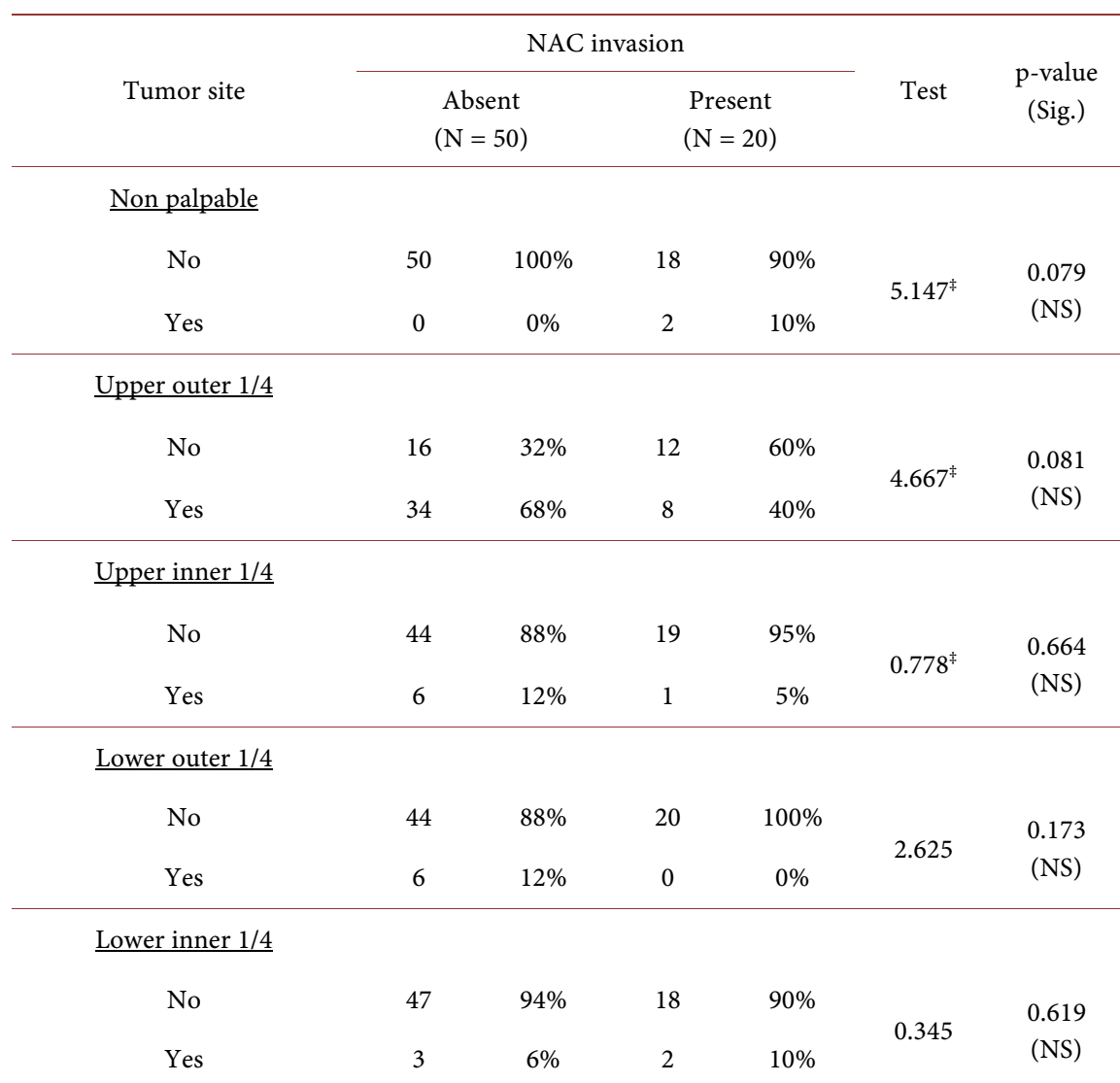

Continued 


\begin{tabular}{ccccccc}
\hline $\begin{array}{c}\text { Central } \\
\text { No }\end{array}$ & 50 & $100 \%$ & 14 & $70 \%$ & & \\
Yes & 0 & $0 \%$ & 6 & $30 \%$ & 16.406 & $\begin{array}{c}<0.001 \\
(\mathrm{HS})\end{array}$ \\
\hline Axillary tail & & & & & & \\
No & 49 & $98 \%$ & 19 & $95 \%$ & & 0.463 \\
Yes & 1 & $2 \%$ & 1 & $5 \%$ & & $(\mathrm{NS})$ \\
\hline
\end{tabular}

${ }^{*}$ Chi-square test. $\mathrm{p}<0.05$ is significant. Sig.: Significance.

Table 4. Size of the tumor $(\mathrm{cm})$ of the studied groups.

\begin{tabular}{ccccc}
\hline & \multicolumn{2}{c}{ NAC invasion } & & \\
\cline { 2 - 3 } Size of the tumor $(\mathrm{cm})$ & $\begin{array}{c}\text { Absent } \\
(\mathrm{N}=50)\end{array}$ & $\begin{array}{c}\text { Present } \\
(\mathrm{N}=20)\end{array}$ & Test & $\begin{array}{c}\text { p-value } \\
\text { (Sig.) }\end{array}$ \\
\hline Clinical & & & & \\
Mean $\pm \mathrm{SD}$ & $3.30 \pm 1.13$ & $2.85 \pm 1.30$ & & 0.401 \\
Median (Range) & $3(1.50-6)$ & $3(0-4.50)$ & & $(\mathrm{NS})$ \\
\hline Ultrasound & & & & \\
Mean \pm SD & $2.60 \pm 1.01$ & $2.57 \pm 0.81$ & & 0.838 \\
Median (Range) & $2.50(1-6)$ & $3(1-4)$ & & (NS) \\
\hline
\end{tabular}

-Mann Whitney U test. $\mathrm{p}<0.05$ is significant. Sig.: Significance.

Table 5. Clinical staging of the studied groups.

\begin{tabular}{|c|c|c|c|c|c|c|}
\hline \multirow[b]{2}{*}{ Clinical staging } & \multicolumn{4}{|c|}{ NAC invasion } & \multirow[b]{2}{*}{ Test } & \multirow{2}{*}{$\begin{array}{c}\text { p-value } \\
\text { (Sig.) }\end{array}$} \\
\hline & \multicolumn{2}{|c|}{$\begin{array}{l}\text { Absent } \\
(\mathrm{N}=50)\end{array}$} & \multicolumn{2}{|c|}{$\begin{array}{l}\text { Present } \\
(\mathrm{N}=11)\end{array}$} & & \\
\hline \multicolumn{7}{|l|}{$\underline{\mathrm{T}}$} \\
\hline $\mathrm{T} 1$ & 10 & $20 \%$ & 1 & $9.1 \%$ & \multirow{4}{*}{$1.002^{\ddagger}$} & \multirow{4}{*}{$\begin{array}{l}0.606 \\
(\mathrm{NS})\end{array}$} \\
\hline $\mathrm{T} 2$ & 39 & $78 \%$ & 10 & $90.9 \%$ & & \\
\hline $\mathrm{T} 3$ & 1 & $2 \%$ & 0 & $0 \%$ & & \\
\hline $\mathrm{T} 4$ & 0 & $0 \%$ & 0 & $0 \%$ & & \\
\hline \multicolumn{7}{|l|}{$\underline{\mathrm{N}}$} \\
\hline No & 20 & $40 \%$ & 0 & $0 \%$ & \multirow{4}{*}{$10.591^{\ddagger}$} & \multirow{4}{*}{$\begin{array}{c}0.014 \\
(\mathrm{~S})\end{array}$} \\
\hline N1 & 15 & $30 \%$ & 3 & $27.3 \%$ & & \\
\hline N2 & 5 & $10 \%$ & 1 & $9.1 \%$ & & \\
\hline N3 & 10 & $20 \%$ & 7 & $63.6 \%$ & & \\
\hline
\end{tabular}

${ }^{\ddagger}$ Chi-square test. $\mathrm{p}<0.05$ is significant. Sig.: Significance.

Table 6. Distance from mass to nipple and depth of the mass in the breast in the studied 
groups.

\begin{tabular}{|c|c|c|c|c|c|c|}
\hline \multirow[b]{2}{*}{ Variables } & \multicolumn{4}{|c|}{ NAC invasion } & \multirow[b]{2}{*}{ Test } & \multirow[b]{2}{*}{$\begin{array}{c}\text { p-value } \\
\text { (Sig.) }\end{array}$} \\
\hline & \multicolumn{2}{|c|}{$\begin{array}{c}\text { Absent } \\
(\mathrm{N}=50)\end{array}$} & \multicolumn{2}{|c|}{$\begin{array}{l}\text { Present } \\
(\mathrm{N}=20)\end{array}$} & & \\
\hline \multicolumn{7}{|c|}{ Distance from mass to nipple } \\
\hline Mean \pm SD & \multicolumn{2}{|c|}{$7.24 \pm 2.73$} & \multicolumn{2}{|c|}{$3.15 \pm 2.80$} & \multirow{2}{*}{$-5.320 \bullet$} & \multirow{2}{*}{$\begin{array}{c}<0.001 \\
(\mathrm{HS})\end{array}$} \\
\hline Median (Range) & \multicolumn{2}{|c|}{$6(4.50-16)$} & \multicolumn{2}{|c|}{$2.75(0-12)$} & & \\
\hline \multicolumn{7}{|l|}{ Depth of the mass } \\
\hline Superficial & 33 & $66 \%$ & 14 & $70 \%$ & \multirow{2}{*}{$0.104^{\ddagger}$} & \multirow{2}{*}{$\begin{array}{l}0.748 \\
(\mathrm{NS})\end{array}$} \\
\hline Deep & 17 & $34 \%$ & 6 & $30 \%$ & & \\
\hline
\end{tabular}

-Mann Whitney U test. ${ }^{\ddagger}$ Chi-square test. $p<0.05$ is significant. Sig.: Significance.

Table 7. Distance from mass to nipple as a predictor for NAC invasion in breast carcinoma; ROC curve analysis.

\begin{tabular}{ccccccc}
\hline $\begin{array}{c}\text { Cut-off } \\
\text { values }\end{array}$ & $\begin{array}{c}\text { SN \% } \\
(95 \% \mathrm{CI})\end{array}$ & $\begin{array}{c}\text { SP \% } \\
(95 \% \mathrm{CI})\end{array}$ & $\begin{array}{c}\text { PPV \% } \\
(95 \% \mathrm{CI})\end{array}$ & $\begin{array}{c}\text { NPV \% } \\
(95 \% \mathrm{CI})\end{array}$ & $\begin{array}{c}\text { Accuracy } \\
(95 \% \mathrm{CI})\end{array}$ & $\begin{array}{c}\text { AUROC } \\
(95 \% \mathrm{CI})\end{array}$ \\
\hline $\mathbf{4} 4 \mathrm{~cm}$ & $\begin{array}{c}85 \% \\
(62.1-96.8)\end{array}$ & $\begin{array}{c}100 \% \\
(92.9-100)\end{array}$ & $\begin{array}{c}100 \% \\
(79.4-100)\end{array}$ & $\begin{array}{c}94.3 \% \\
(84.3-98.8)\end{array}$ & $\begin{array}{c}95.7 \% \\
(84.1-99.1)\end{array}$ & $\begin{array}{c}0.908 \\
(0.815-0964)\end{array}$ \\
\hline
\end{tabular}

${ }^{*} \mathrm{Z}$ test $=5.757$; p-value $<0.001$ (HS). ROC curve: Receiver Operating Characteristic curve; SN: Sensitivity; SP: Specificity; PPV: Positive Predictive Value; NPV: Negative Predictive Value; AUROC: Area Under Receiver Operating Characteristic curve; 95\%CI: 95\% Confidence Interval; $p<0.05$ is significant.

Table 8. Histopathological examination of the studied groups.

\begin{tabular}{|c|c|c|c|c|c|c|}
\hline \multirow[b]{2}{*}{ Histopathological examination } & \multicolumn{4}{|c|}{ NAC invasion } & \multirow[b]{2}{*}{ Test } & \multirow{2}{*}{$\begin{array}{c}\text { p-value } \\
\text { (Sig.) }\end{array}$} \\
\hline & \multicolumn{2}{|c|}{$\begin{array}{l}\text { Absent } \\
(\mathrm{N}=50)\end{array}$} & \multicolumn{2}{|c|}{$\begin{array}{l}\text { Present } \\
(\mathrm{N}=11)\end{array}$} & & \\
\hline \multicolumn{7}{|l|}{ Biopsy type } \\
\hline FNAC & 6 & $12 \%$ & 3 & $27.3 \%$ & & \\
\hline Core biopsy & 32 & $64 \%$ & 7 & $63.6 \%$ & $2.366^{\ddagger}$ & $\begin{array}{c}0.306 \\
\text { (NS) }\end{array}$ \\
\hline Excisional biopsy & 12 & $24 \%$ & 1 & $9.1 \%$ & & \\
\hline \multicolumn{7}{|l|}{$\underline{\text { Histopathology of biopsy }}$} \\
\hline IDC & 42 & $84 \%$ & 10 & $90.9 \%$ & & \\
\hline ILC & 4 & $8 \%$ & 0 & $0 \%$ & $0.944^{\ddagger}$ & $\begin{array}{c}0.624 \\
(\mathrm{NS})\end{array}$ \\
\hline IDC + ILC & 4 & $8 \%$ & 1 & $9.1 \%$ & & \\
\hline \multicolumn{7}{|l|}{ Histopathology of MRM } \\
\hline IDC & 42 & $84 \%$ & 10 & $90.9 \%$ & & \\
\hline ILC & 4 & $8 \%$ & 0 & $0 \%$ & $0.944^{\ddagger}$ & $\begin{array}{c}0.624 \\
(\mathrm{NS})\end{array}$ \\
\hline IDC + ILC & 4 & $8 \%$ & 1 & $9.1 \%$ & & \\
\hline \multicolumn{7}{|l|}{ Histopathology grade } \\
\hline Grade I & 0 & $0 \%$ & 0 & $0 \%$ & $9.350^{\ddagger}$ & \\
\hline
\end{tabular}

\section{Continued}




\begin{tabular}{|c|c|c|c|c|c|c|}
\hline Grade II & 30 & $60 \%$ & 1 & \multicolumn{3}{|l|}{$9.1 \%$} \\
\hline Grade III & 20 & $40 \%$ & 11 & \multicolumn{3}{|l|}{$90.9 \%$} \\
\hline \multicolumn{7}{|l|}{ Lymph node } \\
\hline Node negative & 20 & $40 \%$ & 0 & $0 \%$ & \multirow{2}{*}{$6.546^{\ddagger}$} & \multirow{2}{*}{$\begin{array}{c}0.011 \\
(\mathrm{~S})\end{array}$} \\
\hline Node positive & 30 & $60 \%$ & 11 & $100 \%$ & & \\
\hline \multicolumn{7}{|l|}{$\underline{\text { Positive LN }}$} \\
\hline Mean \pm SD & \multicolumn{2}{|c|}{$8.30 \pm 7.49$} & \multicolumn{2}{|c|}{$13.72 \pm 8.64$} & \multirow{2}{*}{-1.893} & \multirow{2}{*}{$\begin{array}{l}0.058 \\
\text { (NS) }\end{array}$} \\
\hline Median (Range) & \multicolumn{2}{|c|}{$5.50(1-23)$} & \multicolumn{2}{|c|}{$10(2-29)$} & & \\
\hline \multicolumn{7}{|l|}{ Dissected LN } \\
\hline Mean \pm SD & \multicolumn{2}{|c|}{$17.98 \pm 5.38$} & \multicolumn{2}{|c|}{$18.45 \pm 8.58$} & \multirow{2}{*}{$-0.376 \bullet$} & \multirow{2}{*}{$\begin{array}{l}0.707 \\
\text { (NS) }\end{array}$} \\
\hline Median (Range) & \multicolumn{2}{|c|}{$19(5-25)$} & \multicolumn{2}{|c|}{$20(6-32)$} & & \\
\hline
\end{tabular}

${ }^{\ddagger}$ Chi-square test. •Mann Whitney U test. $\mathrm{p}<0.05$ is significant. Sig.: Significance.

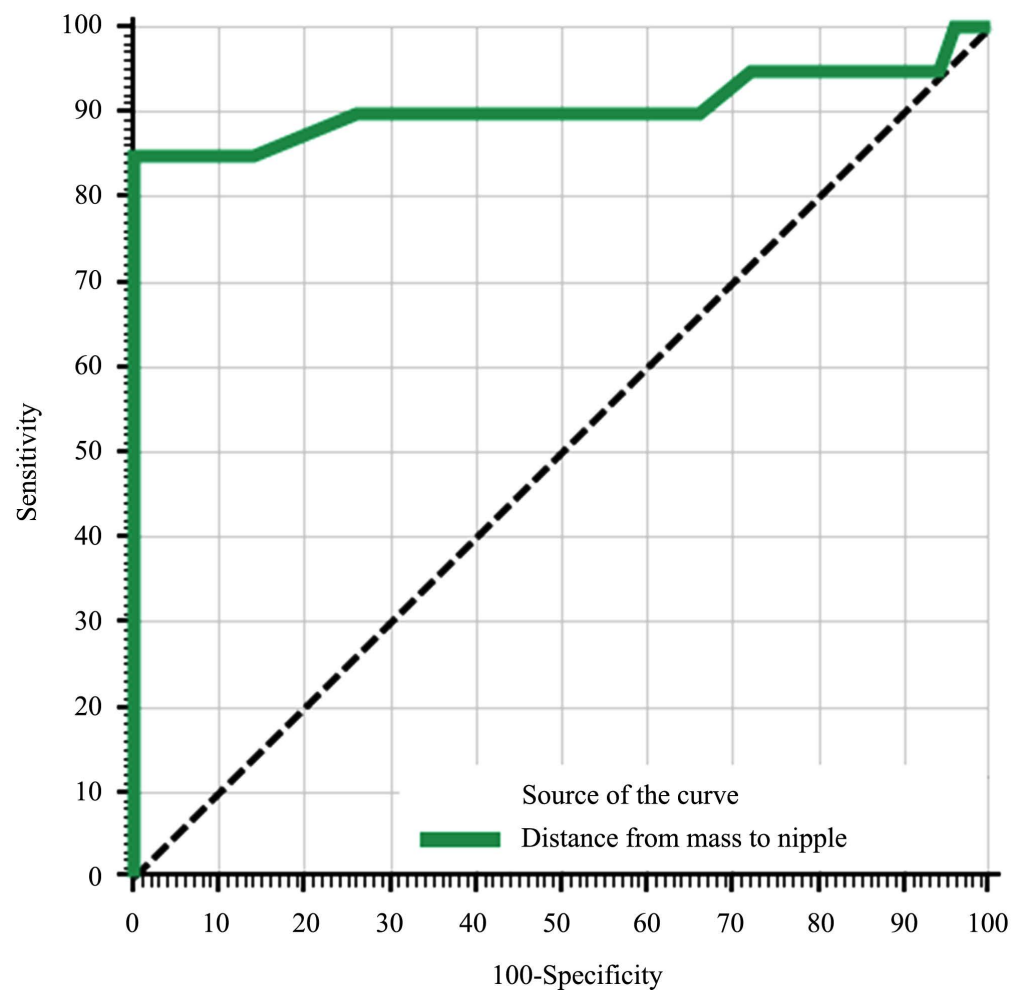

Figure 1. Receiver operating characteristic (ROC) curve of distance from mass to nipple as a predictor for NAC invasion in breast carcinoma.

patients $(90.9 \%)$ with NAC invasion $(\mathrm{p}=0.606)$. There is a significant association between pathological $\mathrm{N}$ stage and NAC invasion where $20 \%$ of patients without NAC invasion had $\mathrm{pN} 3$ versus $63.6 \%$ of patients with NAC invasion $(\mathrm{p}$-value $=0.014) .24 \%$ of patients without NAC invasion had T2N1M0 breast cancer versus $27.3 \%$ in patients with NAC invasion $(\mathrm{p}=$ value $=0.094)$. Stage II constitute $58 \%$ of patients without NAC invasion versus $45.5 \%$ in patients with NAC invasion ( $p$-value $=0.342$ ). There is a significant association between es- 
trogen receptor negativity and NAC invasion where $18 \%$ of patients without NAC invasion had negative estrogen receptor versus $90.9 \%$ of patients with NAC invasion ( $\mathrm{p}$-value $<0.001$ ), also there is a significant association between progesterone receptor negativity and NAC invasion where $24 \%$ of patients without NAC invasion had negative progesterone receptor versus $72.7 \%$ of patients with NAC invasion ( $\mathrm{p}$-value $=0.004$ ), while there is a significant association between HER2 positivity and NAC invasion where $8 \%$ of patients without NAC invasion had positive HER2/neu versus $90.9 \%$ of patients with NAC invasion (p-value <0.001) (Table 9 and Table 10, Figures 2-4).

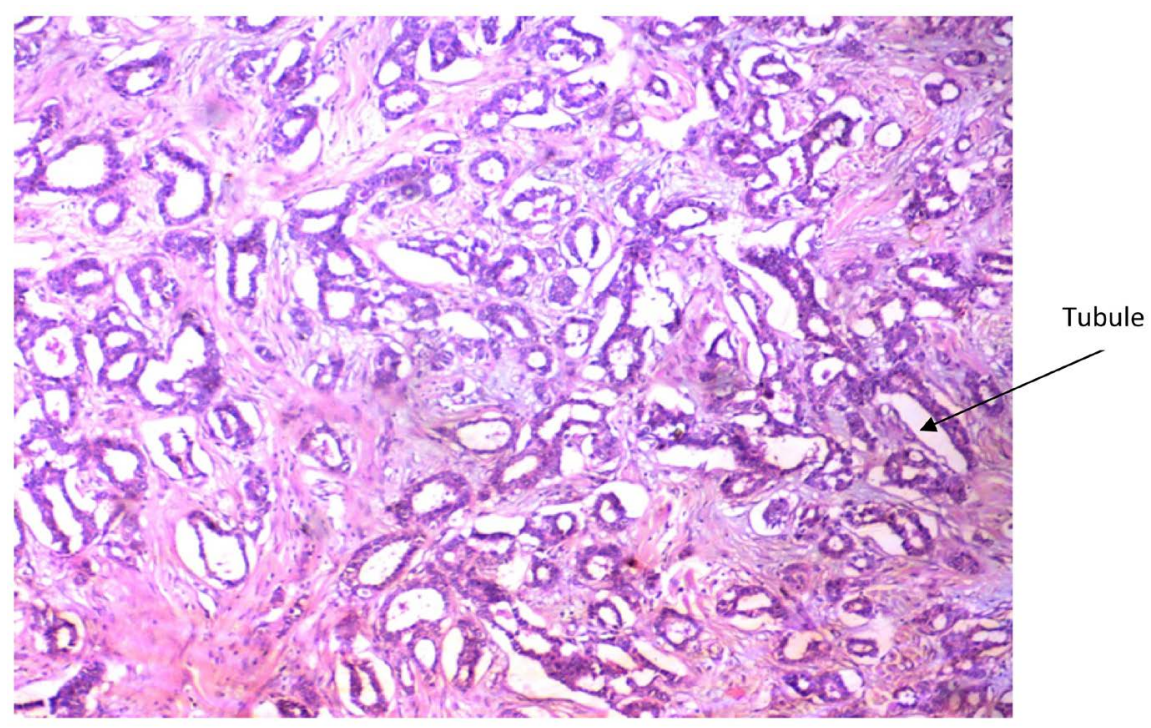

Figure 2. Infiltrating duct carcinoma (IDC) grade II, showing tubular formation $(\mathrm{H} \& \mathrm{E}$ $\times 100)$.

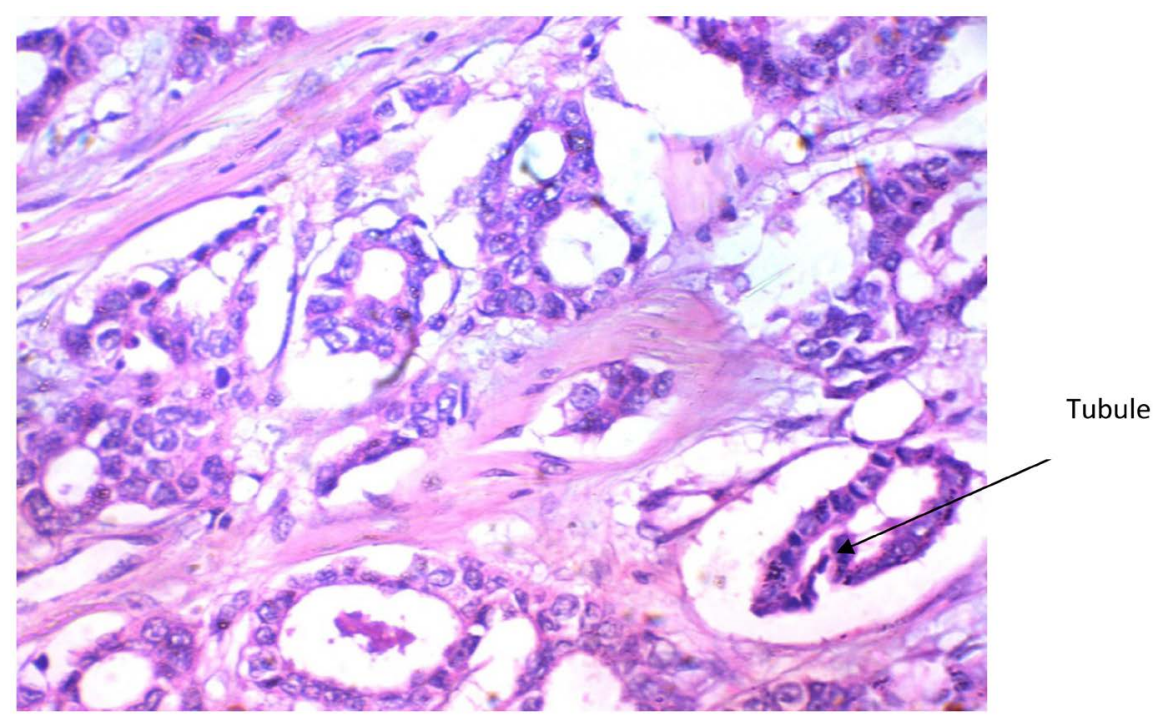

Figure 3. Higher power view of the previous figure showing Infiltrating duct carcinoma (IDC) grade II, showing tubular formation $(\mathrm{H} \& \mathrm{E} \times 400)$.

Table 9. Histopathological staging of the studied groups. 


\begin{tabular}{|c|c|c|c|c|c|c|}
\hline \multirow[b]{2}{*}{ Clinical staging } & \multicolumn{4}{|c|}{ NAC invasion } & \multirow[b]{2}{*}{ Test } & \multirow{2}{*}{$\begin{array}{c}\text { p-value } \\
\text { (Sig.) }\end{array}$} \\
\hline & \multicolumn{2}{|c|}{$\begin{array}{l}\text { Absent } \\
(\mathrm{N}=50)\end{array}$} & \multicolumn{2}{|c|}{$\begin{array}{l}\text { Present } \\
(\mathrm{N}=11)\end{array}$} & & \\
\hline $\mathrm{T} 1$ & 10 & $20 \%$ & 1 & $9.1 \%$ & \multirow{4}{*}{$1.002^{\ddagger}$} & \multirow{4}{*}{$\begin{array}{l}0.606 \\
\text { (NS) }\end{array}$} \\
\hline $\mathrm{T} 2$ & 39 & $78 \%$ & 10 & $90.9 \%$ & & \\
\hline $\mathrm{T} 3$ & 1 & $2 \%$ & 0 & $0 \%$ & & \\
\hline $\mathrm{T} 4$ & 0 & $0 \%$ & 0 & $0 \%$ & & \\
\hline \multicolumn{7}{|l|}{$\underline{\mathrm{N}}$} \\
\hline No & 20 & $40 \%$ & 0 & $0 \%$ & \multirow{4}{*}{$10.591^{\ddagger}$} & \multirow{4}{*}{$\begin{array}{c}0.014 \\
(S)\end{array}$} \\
\hline N1 & 15 & $30 \%$ & 3 & $27.3 \%$ & & \\
\hline $\mathrm{N} 2$ & 5 & $10 \%$ & 1 & $9.1 \%$ & & \\
\hline $\mathrm{N} 3$ & 10 & $20 \%$ & 7 & $63.6 \%$ & & \\
\hline \multicolumn{7}{|l|}{$\underline{\mathrm{TNM}}$} \\
\hline T1N0M0 & 4 & $8 \%$ & 0 & $0 \%$ & \multirow{8}{*}{$12.193^{\ddagger}$} & \multirow{8}{*}{$\begin{array}{l}0.094 \\
(\mathrm{NS})\end{array}$} \\
\hline T2N0M0 & 15 & $30 \%$ & 0 & $0 \%$ & & \\
\hline T1N1M0 & 3 & $6 \%$ & 0 & $0 \%$ & & \\
\hline T2N1M0 & 12 & $24 \%$ & 3 & $27.3 \%$ & & \\
\hline T3N0M0 & 1 & $2 \%$ & 0 & $0 \%$ & & \\
\hline T2N2M0 & 5 & $10 \%$ & 1 & $9.1 \%$ & & \\
\hline T1N3M0 & 3 & $6 \%$ & 1 & $9.1 \%$ & & \\
\hline T2N3M0 & 7 & $14 \%$ & 6 & $54.5 \%$ & & \\
\hline \multicolumn{7}{|c|}{ AJCC stage grouping } \\
\hline Stage I & 4 & $8 \%$ & 0 & $0 \%$ & & \multirow{3}{*}{$\begin{array}{l}0.342 \\
\text { (NS) }\end{array}$} \\
\hline Stage II & 29 & $58 \%$ & 5 & $45.5 \%$ & $2.144^{\ddagger}$ & \\
\hline Stage III & 17 & $34 \%$ & 6 & $54.5 \%$ & & \\
\hline
\end{tabular}

${ }^{\ddagger}$ Chi-square test. $\mathrm{p}<0.05$ is significant. Sig.: Significance.

Table 10. Biological markers of the studied groups.

\begin{tabular}{|c|c|c|c|c|c|c|}
\hline \multirow[b]{2}{*}{ Biological markers } & \multicolumn{4}{|c|}{ NAC invasion } & \multirow[b]{2}{*}{ Test } & \multirow{2}{*}{$\begin{array}{c}\text { p-value } \\
\text { (Sig.) }\end{array}$} \\
\hline & \multicolumn{2}{|c|}{$\begin{array}{c}\text { Absent } \\
(\mathrm{N}=50)\end{array}$} & \multicolumn{2}{|c|}{$\begin{array}{l}\text { Present } \\
(\mathrm{N}=11)\end{array}$} & & \\
\hline Negative & 9 & $18 \%$ & 10 & $90.9 \%$ & \multirow{2}{*}{$22.349^{\ddagger}$} & \multirow{2}{*}{$\begin{array}{c}<0.001 \\
(\mathrm{HS})\end{array}$} \\
\hline Positive & 41 & $82 \%$ & 1 & $9.1 \%$ & & \\
\hline \multicolumn{7}{|l|}{$\underline{\mathrm{PR}}$} \\
\hline Negative & 12 & $24 \%$ & 8 & $72.7 \%$ & \multirow{2}{*}{$9.715^{\ddagger}$} & \multirow{2}{*}{$\begin{array}{c}0.004 \\
(S)\end{array}$} \\
\hline Positive & 38 & $76 \%$ & 3 & $27.3 \%$ & & \\
\hline \multicolumn{7}{|l|}{ HER2/neu overexpression } \\
\hline Negative & 46 & $92 \%$ & 1 & $9.1 \%$ & \multirow{2}{*}{$35.049^{\ddagger}$} & \multirow{2}{*}{$\begin{array}{c}<0.001 \\
(\mathrm{HS})\end{array}$} \\
\hline Positive & 4 & $8 \%$ & 10 & $90.9 \%$ & & \\
\hline
\end{tabular}

${ }^{\ddagger}$ Chi-square test. $\mathrm{p}<0.05$ is significant. Sig.: Significance. 


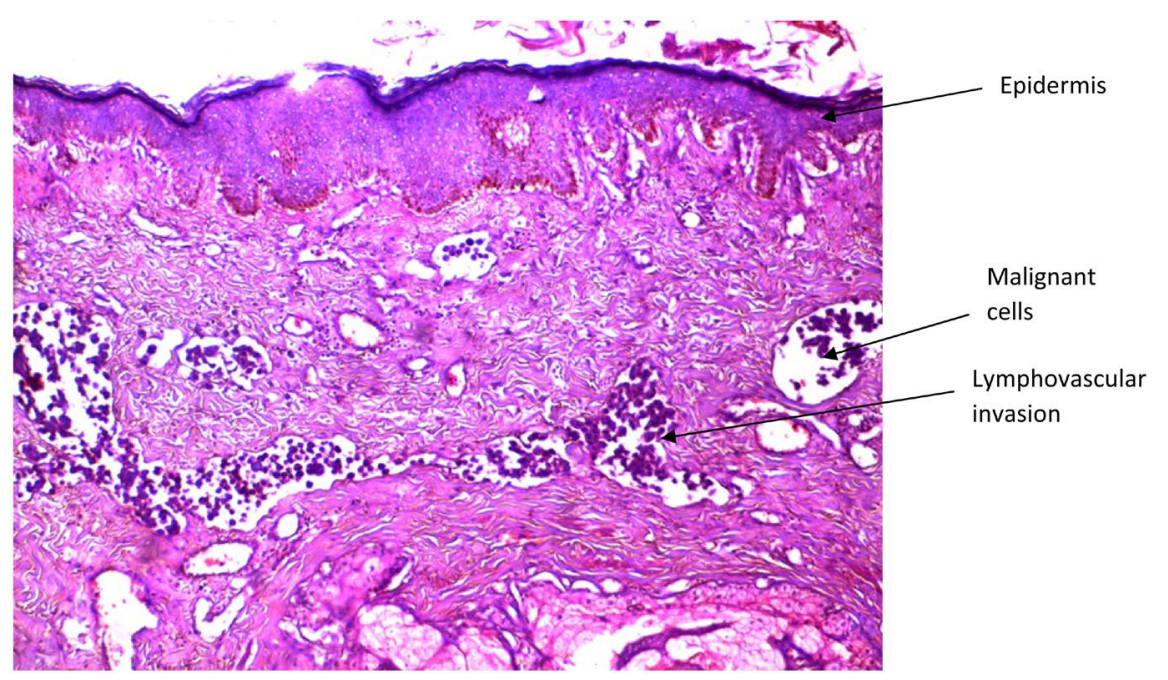

Figure 4. Infiltrating duct carcinoma (IDC) grade III, showing lymphovascular invasion in the subareolar plexuses $(\mathrm{H} \& \mathrm{E} \times 100)$.

\section{Discussion}

Oncoplastic surgery has become a key aspect of breast cancer treatment, as it considers both oncological and cosmetic outcomes. The idea of sparing the skin of the breast is to facilitate the immediate breast reconstruction [6].

The greatest advantage of nipple areola sparing mastectomy compared to skin sparing mastectomy is the immediate cosmetic result with no need for nipple reconstruction later on. The need to remove the nipple areola complex is based on the concern of occult neoplastic involvement [7].

The concept of the nipple areola sparing mastectomy was popularized by Freeman in the 1960's and 1970's, the procedure was referred to as a subcutaneous mastectomy. The use of NAS mastectomy for the treatment of breast cancer grew in the 1970's and 1980's. The attempts are made to preserve the nipple areola complex since the first paper done by Gerber et al in 2003 [8].

In this study, we evaluated the oncological safety for nipple areola sparing mastectomy procedure by searching for the factors that predict the presence of nipple areola complex invasion. This done by history taking and clinical examination of 60 Egyptian female patients with breast cancer. Then we searched for the presence of the occult malignant cells in the subareolar tissue in the breast specimens of the standard modified radical mastectomy (done by single expert pathologist) for the 60 patients.

In this study, NAC invasion was noted in 9 out of 60 mastectomy specimens. So, the incidence occult NAC invasion was $15 \%$. This rate indicates that even patients who had clinically normal-appearing NAC should be carefully selected for NAS mastectomy. This falls in the same range reported by Gomez et al., (2014) who reported that the incidence of nipple involvement (NI) ranging from $0 \%$ to $58 \%$ [9].

In our study, the mean age of breast cancer patients without NAC invasion was non-significantly higher than mean age of breast cancer patients with NAC 
invasion (Mean: 51.26 vs 49.90 years, p-value $=0.741$ ) There was statistically non-significant association between age groups and NAC invasion in which (p-value $=0.121)$ and this coincides with Zhang et al., (2015) [10].

In this study, premenopausal women were $22(36.7 \%)$ and postmenopausal were $38(63.3 \%)$. There was statistically non-significant association between menstrual state and NAC invasion, where $64 \%$ of patients without NAC invasion were postmenopausal vs $54.5 \%$ in patients with NAC invasion ( $\mathrm{p}$-value $=$ 0.558). This coincides with Abou Nagah and El-Sabaa, (2012) [11].

In this study, 13 out of 50 patients (26\%) without NAC invasion had positive family history of breast cancer versus 3 out of 11 patients (27.3\%) with NAC invasion. There was statistically non-significant association between family history and NAC invasion in which ( $\mathrm{p}$-value $=1.000$ ) and this differs with other studies that reported that up to $5 \%-10 \%$ of breast cancer are due to genetic mutations [12].

In this study, the most common patient's complaints were painless lump (58.3\%), painful lump (26.7\%) and nipple retraction (8.3\%). There was a statistically significant association between nipple retraction and NAC invasion where $2 \%$ of patients without NAC invasion complaint from nipple retraction versus $36.7 \%$ of patients with NAC invasion ( $p$-value $=0.003$ ).

Other patient's complaints had statistically nonsignificant association with NAC invasion as painless lump ( $\mathrm{p}$-value $=0.174)$, painful lump ( $\mathrm{p}$-value $=$ $0.711)$, axillary lump ( $\mathrm{p}$-value $=0.500)$ and bleeding per nipple $(\mathrm{p}$-value $=0.180)$.

In this study, there was no statistically significant association between breast size (measured by the bra size) and NAC invasion as mean breast size in patients without NAC invasion was non-significantly lower than mean breast size in patients with NAC invasion ( $\mathrm{p}$-value $=0.186$ ) and this coincides with Abou Nagah and El-Sabaa, (2012) [11].

In this study, there was a statistically highly significant association between focality and NAC invasion where none of patients without NAC invasion had multifocal breast cancer versus $81.8 \%$ of patients with NAC invasion (p-value < 0.001). This coincides with Zhang et al., (2015) [10].

Wang et al., (2012) and Weidong et al., (2011) who suggested that patients with multifocal or multicentric tumors are at higher risk to get nipple involvement and differs with Brachtel et al., (2009) who found no significant association between multifocal tumors and NAC invasion [13] [14] [15].

In this study, the upper outer quadrant was the most affected one (42 patients with $60 \%$ incidence). This matches with Skandalakis et al., (2016) who reported that the upper outer quadrant contains the main bulk of breast tissue and thus it is the most usual site for both breast cancer and most benign breast pathologies [16].

There was a highly significant association between tumor site (centrally located tumors) and NAC invasion, as tumors located in the central areas are more likely to have nipple invasion (NI) than peripheral areas ( $\mathrm{p}$-value $<0.001$ ). This matches with Simmons et al., (2019). Other tumor sites have nonstatistical- 
ly significant association with NAC invasion as the upper outer quadrant ( $\mathrm{p}$-value $=0.081)$, upper inner quadrant $(\mathrm{p}$-value $=0.664)$, lower outer quadrant $(\mathrm{p}$-value $=0.173)$, lower inner quadrant $(\mathrm{p}$-value $=0.619)$ and axillary tail $(\mathrm{p}$-value $=0.493)[17]$.

In this study, there was no statistically significant association between tumor size neither clinically assisted or US assisted and NAC invasion ( $p$-value $=0.401$ ) ( $\mathrm{p}$-value $=0.838)$. This matches with Lawrence et al., (2018) whose results failed to show any statistically significant association between tumor size and occult nipple involvement, and this differ with Zhang et al., (2015) who reported that the risk of NAC invasion increased significantly in patients with larger tumors [10] [18].

In this study, there was statistically highly significant association between the tumor nipple distance and NAC invasion (p-value $<0.001$ ). This reported by Weidong et al., (2017) [13].

In our study, the optimum cutoff of distance from mass to nipple as a predictor for NAC invasion in breast carcinoma was $\leq 4 \mathrm{~cm}$. So, all of patients with distance $>4 \mathrm{~cm}$ can be expected that they have not NAC invasion. So, we can conclude that a distance $>4 \mathrm{~cm}$ is requested for NAS mastectomy. This coincides with Vlajcic et al., (2016) who found that the NAC could be safely preserved with tumor-to-nipple distance $>4 \mathrm{~cm}$ and does not match with Zhang et al., (2015) that suggested distance of $2.5 \mathrm{~cm}$ from the tumor to the nipple is required to reduce the risk of nipple involvement [10] [19].

In our study, there was no statistically significant association between histological type of the tumor and NAC invasion ( $\mathrm{p}$-value $=0.624$ ). This agrees with Zhang et al., (2015) [10].

In this study, there was a statistically significant association between histological grade of the tumor and NAC invasion, where $40 \%$ of patients without NAC invasion was grade III versus $90.9 \%$ of patients with NAC invasion ( $\mathrm{p}$-value = 0.002). This coincides with Eisenberg et al., (2014), but differs with Simmons et al., (2019) who found no significant association in rates of NAC invasion and tumor grades [17] [20].

In this study, there was a statistically significant association between positive lymph node invasion and nipple invasion as $60 \%$ of patients without NAC invasion were node positive versus $100 \%$ of patients with NAC invasion ( $\mathrm{p}$-value = 0.011). Number of positive lymph node in patients with NAC invasion was significantly higher than in patients without NAC invasion ( $p$-value $=0.001$ ). This coincides with study who suggested that NAC invasion was significantly increased in patients with lymph node metastasis and differs with Simmons et al., (2019) their study did not show a higher incidence of NAC invasion in lymph node positive group [17] [21].

In our study, there was a statistically highly significant association between estrogen receptor negativity and NAC invasion where $18 \%$ of patients without NAC invasion had negative estrogen receptor versus $90.9 \%$ of patients with NAC invasion ( $p$-value $<0.001$ ). Also, there was a statistically significant associ- 
ation between progesterone receptor negativity and NAC invasion where $24 \%$ of patients without NAC invasion had negative progesterone receptor versus $72.7 \%$ of patients with NAC invasion (p-value $=0.004$ ). This agrees with Zhang et al., (2015) who reported that the trend toward higher nipple involvement rate was found in patients with negative ER status and negative PR status [10].

In our study, there was a statistically highly significant association between HER2 positivity and NAC invasion where $8 \%$ of patients without NAC invasion had positive HER2 versus $90.9 \%$ of patients with NAC invasion (p-value < 0.001). This agrees with Zhang et al., (2015) who suggested that patients with positive (HER2) have a higher rate of NAC invasion [10].

In our study, we found that the tumor site and tumor nipple distance affect the prediction of NAC invasion while the age, menstrual state, breast size and tumor type did not directly affect the prediction of presence of subareolar malignant cells. This coincides with Gulben et al., (2009) and differs with Brachtel et al., (2019) who found that tumor size affects NAC invasion [14] [22].

According to our study the ideal patients for NAS mastectomy should have these criteria: clinically normal nipple areola complex, tumor nipple distance is $>4 \mathrm{~cm}$, No multi-centric tumor, absence of lymph node involvement, peripheral not central tumor and absence of sub-areolar lymphovascular invasion (LVI). This coincides with Simmons et al., (2019) [17].

\section{Conclusions}

According to our study, the ideal patients for NAS mastectomy should have these criteria:

1) Clinically normal nipple areola complex.

2) Distance from the tumor to the nipple is $>4 \mathrm{~cm}$.

3) No multifocal/multicentric tumor.

4) Absence of lymph node invasion.

5) Tumor grade (grade I, II).

6) Peripheral not central tumor.

7) No sub-areolar lymphovascular invasion (LVI).

8) ER receptor positive.

9) PR receptor positive.

10) HER2 negative.

So, we recommend taking these criteria in preoperative decision making for NAS mastectomy.

\section{Conflicts of Interest}

The authors declare no conflicts of interest regarding the publication of this paper.

\section{References}

[1] Adrada, B., Arribas, E., Gilcrease, M. and Yang, W.T. (2019) Invasive Micropapil- 
lary Carcinoma of the Breast: Mammographic, Sonographic and MRI Features. American Journal of Roentgenology, 193, W58-W63.

[2] Agrawal, A., Sibbering, D.M. and Courtney, C.A. (2013) Skin Sparing Mastectomy and Immediate Breast Reconstruction: A Review. European Journal of Surgical Oncology, 39, 320-328. https://doi.org/10.1016/j.ejso.2012.12.015

[3] Agur, A.M.R. and Dalley, A.F. (2018) The Breast. In: Agur, A.M.R. and Dalley, A.F., Eds., Grant's Atlas of Anatomy, Vol. 1, 12th Edition, Lippincott Williams \& Wilkins, Philadelphia, 5-9.

[4] Alperovich, M., Tanna, N., Samra, F., Blechman, K., Shapiro, R.L., Guth, A.A., et al. (2013) Nipple-Sparing Mastectomy in Patients with a History of Reduction Mammaplasty or Mastopexy: How Safe Is It? Plastic and Reconstructive Surgery, 131, 962-967. https://doi.org/10.1097/PRS.0b013e3182865ad2

[5] Araco, A., Gravante, G., Araco, F., Gentile, P., Castrì, F., Delogu, D., et al. (2016) Breast Asymmetries: A Brief Review and Our Experience. Aesthetic Plastic Surgery, 30, 309-319. https://doi.org/10.1007/s00266-005-0178-x

[6] Rose, M., Manjer, J., Ringberg, A. and Svensson, H. (2014) Surgical Strategy, Methods of Reconstruction, Surgical Margins and Postoperative Complications in Oncoplastic Breast Surgery. European Journal of Plastic Surgery, 37, 205-214. https://doi.org/10.1007/s00238-013-0922-4

[7] Skotnicki, P., Ryś, J., Blecharz, P., Reinfuss, M., Jakubowicz, J., Ambicka, A., et al. (2012) Invasive Lobular Carcinoma of the Breast: Cytometric and Immunohistochemical Characteristics of 96 Cases. Polish Journal of Pathology, 63, 112-120.

[8] Harness, J.K., Vetter, T.S. and Salibian, A.H. (2011) Areola and Nipple-Areola-Sparing Mastectomy for Breast Cancer Treatment and Risk Reduction: Report of an Initial Experience in a Community Hospital Setting. Annals of Surgical Oncology, 18, 917-922. https://doi.org/10.1245/s10434-010-1365-9

[9] Gomez, C., Shah, C., Mc Closkey, S., Foster, N. and Vicini, F. (2014) The Role of Radiation Therapy after Nipple-Sparing Mastectomy. Annals of Surgical Oncology, 21, 2237-2244. https://doi.org/10.1245/s10434-013-3446-Z

[10] Zhang, H., Moran, M., Haffty, B., Haffty, B.G. and Yang, Q. (2015) Predictive Factors of Nipple Involvement in Breast Cancer: A Systematic Review and Meta-Analysis. Breast Cancer Research and Treatment, 151, 239-249.

https://doi.org/10.1007/s10549-015-3385-4

[11] Abou Nagah, G. and El-Sabaa, B. (2012) Nipple-Areola and Skin Sparing Mastectomy: Is it Oncologically Safe Procedure in Egyptian Females. Egyptian Journal of Surgery, 31, 12-15.

[12] Sinn, H.P. and Kreipe, H. (2013) A Brief Overview of the WHO Classification of Breast Tumors, 4th Edition, Focusing on Issues and Updates from the 3rd Edition. Breast Care, 8, 149-154. https://doi.org/10.1159/000350774

[13] Weidong, L., Wang, S., Guo, X., Lang, R., Fan, Y., Gu, F., et al. (2017) Nipple Involvement in Breast Cancer Retrospective Analysis of 2323 Consecutive Mastectomy Specimens. International Journal of Surgical Pathology, 19, 328-334. https://doi.org/10.1177\%2F1066896911399279

[14] Brachtel, E.F., Rusby, J.E., Michaelson, J.S., Leon Chen, L., Muzikansky, A., Smith, B.L., et al. (2009) Occult Nipple Involvement in Breast Cancer: Clinicopathologic Findings in 316 Consecutive Mastectomy Specimens. Journal of Clinical Oncology, 27, 4948-4954. https://doi.org/10.1200/JCO.2008.20.8785

[15] Wang, J., Xiao, X., Iqbal, N., Iqbal, N., Baxter, L., Skinner, K.A., et al. (2012) Predictors of Nipple-Areolar Complex Involvement by Breast Carcinoma: Histopatho- 
logic Analysis of 787 Consecutive Therapeutic Mastectomy Specimens. Annals of Surgical Oncology, 19, 1174-1180. https://doi.org/10.1245/s10434-011-2107-3

[16] Skandalakis, J., Colborn, G.L., Skandalakis, P.N., et al. (2016) Breast. In: Skandalakis, J., Colborn, G.L., Skandalakis, L.J. and Weidman, T.A., Eds., Skandalakis Surgical Anatomy. The Embryologic and Anatomic Basis of Modern Surgery, PMP, Athens, 106-107.

[17] Simmons, R.M., Brennan, M., Christos, P., King, V. and Osborne, M. (2019) Analysis of Nipple/Areolar Involvement with Mastectomy: Can the Areola Be Preserved? Annals of Surgical Oncology, 9, 165-168.

https://doi.org/10.1007/BF02557369

[18] Lawrence, R.A. and Lawrence, R.M. (2018) Breastfeeding: A Guide for the Medical Profession, 6th Edition, Elsevier, Philadelphia, 39-64.

[19] Vlajcic, Z., Zic, R., Stanec, S., Lambasa, S., Petrovecki, M. and Stanec, Z. (2016) Nipple-Areola Complex Preservation: Predictive Factors of Neoplastic Nipple-Areola Complex Invasion. Annals of Plastic Surgery, 55, 240-244.

https://doi.org/10.1097/01.sap.0000171680.49971.85

[20] Eisenberg, R.E., Chan, J.S., Swistel, A.J. and Hoda, S.A. (2014) Pathological Evaluation of Nipple Sparing Mastectomies with Emphasis on Occult Nipple Involvement: The Weill-Cornell Experience with 325 Cases. The Breast Journal, 20, 15-21. https://doi.org/10.1111/tbj.12199

[21] Endara, M., Chen, D., Verma, K., Nahabedian, M.Y. and Spear, S.L. (2013) Breast Reconstruction Following Nipple-Sparing Mastectomy: A Systematic Review of the Literature with Pooled Analysis. Plast Reconstr Surg, 132, 1043-1054. https://doi.org/10.1097/PRS.0b013e3182a48b8a

[22] Gulben, K., Yildirim, E. and Berberoglu, U. (2009) Prediction of Occult Nipple-Areola Complex Involvement in Breast Cancer Patients. Neoplasma, 56, 72-75. https://doi.org/10.4149/neo $200901 \quad 72$ 